\title{
Characterization of bacterial communities of rhizosphere and rhizoplane of Early Zhukovsky potato
}

\author{
Marat Lutfulin ${ }^{1, *}$, Darya Zaripova ${ }^{1}$, Oksana Moiseeva ${ }^{1}$, Semen Vologin ${ }^{2}$, and Ayslu \\ Mardanova $^{1}$ \\ ${ }^{1}$ SRL "Microbial biotechnologies", Institute of Fundamental Medicine and Biology, Kazan Federal \\ University, Kazan, Russia \\ ${ }^{2}$ Tatar Research Institute of Agriculture, Kazan, Russia
}

\begin{abstract}
Identification of patterns of formation of bacterial communities of the rhizosphere and rhizoplane of potato (Solanum tuberosum L.), the most important agricultural crop, is necessary for the introduction and maintenance of sustainable organic farming. The purpose of this work was the study of the biodiversity of the bacterial microbiota of the rhizosphere and rhizoplane of Early Zhukovsky potato, cultivated on gray forest soils. Comparative analysis based on sequencing of the $16 \mathrm{~S}$ R RNA gene showed a significant difference in the representation of different groups of bacteria in these potato root compartments. Thus, the proportions of the dominant bacteria in the rhizosphere and rhizoplane of the Proteobacteria phylum reach $47.66 \% \pm 7.22 \%$ and $86.35 \% \pm 0.53 \%$, respectively $(P<$ 0.05). In contrast, the representation of phylum Bacteroidetes and Firmicutes in the rhizosphere is significantly higher and reaches $41.45 \% \pm$ $10.42 \%$ and $6.49 \% \pm 3.23 \%$, respectively, compared to the rhizoplane $(7.84 \% \pm 1.24 \%$ and $0.43 \% \pm 0.48 \%,(P<0.05)$. At the same time, Actinobacteria phylum bacteria are present in both compartments in approximately equal amounts $(4.40 \% \pm 1.81 \%$ in the rhizosphere and 5.37 $\% \pm 1.42 \%$ in the rhizoplane). Thus, it was found that potato forms different bacterial communities in the rhizosphere and rhizoplane in quantitative proportions, which is probably determined by the functional role of these microorganisms in the plant physiology.
\end{abstract}

\section{Introduction}

The plant microbiota integrates the microbial community of the rhizosphere, rhizoplane, phyllosphere and endosphere zone of plants [1]. The number of microorganisms in the phyllosphere zone can reach $10^{5}-10^{6} \mathrm{CFU} / \mathrm{cm}^{2}$ of the leaf, while in the root zone $-10^{8}-10^{9}$ $\mathrm{CFU} / \mathrm{g}$ of soil. In the near-root zone of plants, the greatest number of microorganisms is present, and it is the soil that is the reservoir of microbes for the plant microbiome formation [2], and the rhizosphere is one of the most complex ecosystems on Earth [3].

\footnotetext{
* Corresponding author: lutfullin.marat2012@yandex.ru
} 
Thus, the near-root zone is a special niche consisting of a more complex microbial community than other plant organs. The root zone microbiota includes bacteria, actinobacteria, archaea, and fungi $[4,5]$ and is specifically formed for each plant species, playing a huge role in the formation of their productivity [6]. The microbial community of plants, as a whole organism, directly affects the host in several ways: it supplies the plant with nutrients by fixing atmospheric nitrogen or solubilization of phosphorous compounds, stimulates plant growth by relieving stress through the production or degradation of phytohormones, supports plant health by competing with pathogenic microorganisms and inducing plant resistance [7]. The structure of the microbial community is complex and its role in plant physiology has not yet been sufficiently studied. Currently, more and more scientists consider the plant and its microflora as a whole, for which the term holobiont is proposed [8]. It is believed that microorganisms that colonize various plant organs and tissues contain the second genome of the plant [9]. In this regard, interactions between microorganisms and plants that ensure plant health and can ensure sustainable crop production in changing agricultural conditions are actively studied [10].

It is known that the interaction of microorganisms with roots is a key factor determining plant productivity: useful rhizosphere microbes change the morphology of plants, increase their growth and stability, increase the content of mineral substances [11], and also, forming biofilms, inhibit soil pathogens [16]. The bacterial microbiota of the rhizosphere is higher in number, but poorer in diversity of free-soil microbiota species, which indicates the ability of plants to create a selective ecological niche for specific microorganisms [12]. Different plant species form differentiated microbial communities of the rhizosphere, releasing various metabolites into the root zone $[13,14]$. In general, the interaction of plants with microorganisms is established in a complex form due to numerous chemical bonds. Changes in the environment lead to the expression of certain plant genes and the synthesis of a huge number of primary and secondary metabolites, which affects the formation of certain microbial communities $[15,16]$. Research in the field of metabolomics has made it possible to identify metabolites of root exudates (organic substances released in the process of vital activity by the plant root system) and to establish their role in the formation of the microbial community of various root compartments [17, 18]. As it is known, microorganisms of the near-root zone are divided into two groups: the microflora of the rhizoplane (inhabiting the root surface) and the microflora of the rhizosphere (living in the soil directly adjacent to the root). The microflora of the rhizoplane is formed under the direct influence of the plant itself and differs in quantitative and qualitative composition from the microflora of the rhizosphere, the diversity of which largely depends on the type of soil, $\mathrm{pH}$, and nutrients of the host plant [2].

Modern molecular genetic methods based on the use of sequencing methods allow to study in more detail the microbial communities of various plants, to establish the full range of not only cultivated, but also uncultivated representatives. Knowledge of the patterns of plant microbiota formation is important for understanding the role of microorganisms in plant growth, development and protection, as well as for increasing the productivity of practically important crops. Thus, it is important to study and characterize the root microbiota of important agricultural crops.

The purpose of this work was the comparative analysis of bacterial communities of the rhizosphere and rhizoplane of Early Zhukovsky potato with the use of high-throughput sequencing.

\section{Materials and methods}

\subsection{The selection of potato roots}


Samples of potato roots of the Early Zhukovsky variety were used provided by employees of TatSRIAC "NIVA" (Tatarstan, coordinates of the selection point - 55 $38^{\prime}$ n.w. and $49^{\circ} 18^{\prime}$ e.1.). The location of the sampling points was recorded using the GPS system. Potato plants were grown in gray forest soils for 35 days. To isolate the total DNA of the potato rhizosphere and rhizoplane, samples of healthy plant roots were selected in 6 field sections with 4 potato bushes for each repetition. The roots were placed in sterile falcons and stored at $-80^{\circ} \mathrm{C}$ before DNA isolation. To determine the mass of soil contained on the root surface, the roots were weighed immediately after separation from the soil and after washing. Thus, 6 samples of rhizosphere soil and 6 samples of rhizoplane were prepared for sequencing.

\subsection{DNA Isolation}

Samples of potato rhizosphere and rhizoplane for total DNA isolation were prepared according to the method described in work [19]. To isolate bacteria from the rhizosphere, non-rhizospheric soil was removed from the roots by shaking, $5 \mathrm{~g}$ of roots were selected, which were placed in a sterile PBS buffer $(50 \mathrm{ml})$ and intensively shaken for $1 \mathrm{~min}$ until a homogeneous soil suspension was obtained. $2 \mathrm{ml}$ of the soil suspension was centrifuged at $12,000 \mathrm{rpm}$ for $2 \mathrm{~min}$, the supernatant was drained, and the residue was used to isolate the total rhizosphere DNA. To isolate the rhizoplane DNA, the roots were washed 3 times with a sterile PBS buffer after removal of the rhizosphere soil, and then three-time ultrasonic treatment (Branson Unltrasonics) was used for 30 seconds at $4{ }^{\circ} \mathrm{C}$ at $50-60 \mathrm{~Hz}$ to remove all closely adjacent microorganisms from the root surface. The resulting microbial suspensions were filtered through sterile membrane filters with a pore diameter of 0.22 microns (CAMEO ${ }^{\circ}$, GVS, Italy), which were homogenized to isolate the total rhizoplane DNA. DNA was isolated using commercial FastDNA ${ }^{\circledR}$ SPIN Kit for Soil according to the protocol. The average DNA concentration was $18 \mathrm{ng} / \mathrm{ml}$.

\subsection{Analysis of the bacterial microbiota of the potato rhizosphere and rhizoplane}

DNA was used as a matrix in the PCR reaction with universal primers to the conserved region of the 16S rRNA gene (For: 5'-GAGTTTGATCCTGGCTCAG, Rev: 5'ACGGTTACCTTGTTACGAC TT) with the addition of oligonucleotide identifiers for each sample. Sample preparation and sequencing were performed on the Illumina MiSeq device according to the manufacturer's recommendations for the paired-end method.

\subsection{Bioinformatic analysis}

Bioinformatic analysis of sequenced nucleotide sequences of the 16S rRNA gene was performed using the QIIME program (version 13_8). The taxonomic structure of the community was assessed by the OTE shares assigned to different taxa.

\subsection{Statistical analysis}

Statistical analysis was performed using two-way ANOVA analysis and Student's tcriterion. 


\section{Results and discussion}

A comparative analysis was performed based on sequencing of the $16 \mathrm{~S}$ rRNA gene sequences of bacterial communities of the rhizosphere and rhizoplane of potato plants of the Early Zhukovsky variety grown on gray forest soils in the Republic of Tatarstan.

The microbial community of the rhizosphere of all samples was dominated by bacteria of the Proteobacteria, Bacteroidetes, Actinobacteria, and Firmicutes phylum. In the rhizosphere and rhizoplane representatives of the Proteobacteria phylum account for $47.66 \% \pm 7.22 \%$ and $86.35 \% \pm 0.53(P<0.05)$, respectively. The representation of the Bacteroidetes phylum in the rhizoplane was 5 times lower $(7.84 \% \pm 1.24 \%)$ compared to the rhizosphere $(41.45 \% \pm 10.42 \%)(P<0.05)$. Also, the proportion of Firmicutes phyla in the rhizosphere is higher $(6.49 \% \pm 3.23 \%)$ compared to rhizoplane $(0.43 \% \pm 0.48 \%)(P<$ $0.05)$. The proportion of representatives of the Actinobacteria phylum was at the same level and was $4.40 \% \pm 1.81 \%$ in the rhizosphere and $5.37 \% \pm 1.42 \%$ in the rhizoplane. It is known that representatives of this bacteria phylum actively participate in the synthesis of various secondary metabolites [20].

The Proteobacteria phylum is represented in root compartments by two main classes: Alphaproteobacteria and Gammaproteobacteria. Previously, it was shown that a high percentage of these representatives indicates the fertility of rhizosphere soils [21]. The proportion of Alphaproteobacteria representatives was 4 times higher in the potato rhizosphere $(23.75 \% \pm 2.51 \%)$ compared to rhizoplane $(6.93 \% \pm 5.20 \%)(P<0.05)$. On the contrary, representatives of the Gammaproteobacteria class dominated in the rhizoplane, the proportion of which reached $79.41 \% \pm 5.55 \%$, which is significantly higher than the representation of these bacteria in the rhizosphere $(28.21 \% \pm 4.04 \%)(P<0.05)$. The proportion of Betaproteobacteria representatives in the potato rhizosphere and rhizoplane did not exceed $0.02 \%$ and did not differ significantly.

The classes of Sphingobacteriia and Flavobacteriia included in the Bacteroidetes phylum were represented in the rhizosphere in the amount of $6.31 \% \pm 1.79 \%$ and $30.97 \%$ $\pm 6.85 \%$, respectively, which is 4 times higher compared to the rhizoplane $(1.75 \% \pm$ $0.71 \%$ and $6.09 \% \pm 1.21 \%)(P<0.05)$. Firmicutes phylum is represented in both the rhizosphere and rhizoplane by almost one class of Bacilli, the proportion of which was higher in the rhizosphere $5.44 \% \pm 2.18 \%$ compared to the rhizoplane $(0.43 \% \pm 0.48 \%)(P$ $<0.05)$. Actinobacteria phylum is also represented in root compartments by one class of Actinobacteria, the content of which in the rhizosphere was $4.40 \% \pm 1.81 \%$, and in the rhizoplane $-5.37 \% \pm 1.42 \%$.

Figure 1 shows the bacterial genera dominating potato roots and, as can be seen from the diagrams, the rhizosphere and rhizoplane samples differ significantly in the representation of different groups of bacteria. At the level of genera, the dominant representatives of Bacteroidetes were Flavobacterium (rhizosphere - 20.18 \% $\pm 3.27 \%$, rhizoplane $-1.72 \% \pm 1.84 \%(P<0.05)$ ), Chryseobacterium (rhizosphere $-10.75 \% \pm$ $5.59 \%$, rhizoplane $-4.38 \% \pm 2.50 \%(P<0.05)$ ) and Pedobacter (rhizosphere $-6.31 \% \pm$ $1.79 \%$, rhizoplane $-1.75 \% \pm 0.71 \%$ ) (Fig. 1). The Bacillus genus (Firmicutes phylum) is represented higher in the rhizosphere $(6.01 \% \pm 2.49 \%)$ than in the rhizoplane $(0.43 \% \pm$ $0.48 \%$ ). The proportions of bacteria of the Kaistobacter and Sphingomonas genera, representatives of the class of Sphingobacteriia, were also significantly higher in the rhizosphere $(6.76 \% \pm 1.56 \%$ and $7.58 \% \pm 0.96 \%)$ than in the rhizoplane $(0.12 \% \pm 0.10$ $\%$ and $0.50 \% \pm 0.45 \%)(P<0.05)$. On the other hand, the representation of Pseudomonas bacteria belonging to the Gammaproteobacteria class was higher in the rhizoplane $(13.52 \%$ $\pm 5.37 \%)$ compared to the rhizosphere $(5.82 \% \pm 0.80 \%)$, as well as Citrobacter bacteria $(16.33 \% \pm 8.02 \%$ in the rhizoplane; $8.06 \% \pm 3.23 \%$ in the rhizosphere) and representatives of Stenotrophomonas genus of the Xanthomonadaceae family (rhizoplane - 
$17.89 \% \pm 6.05 \%$, rhizosphere $-4.86 \% \pm 2.38 \%)(P<0.05)$. The proportion of representatives of Agrobacterium of the Rhizobiaceae family was almost identical in the rhizosphere and in the rhizoplane and reached $5.96 \% \pm 1.41 \%$ and $3.73 \% \pm 3.47$, respectively.

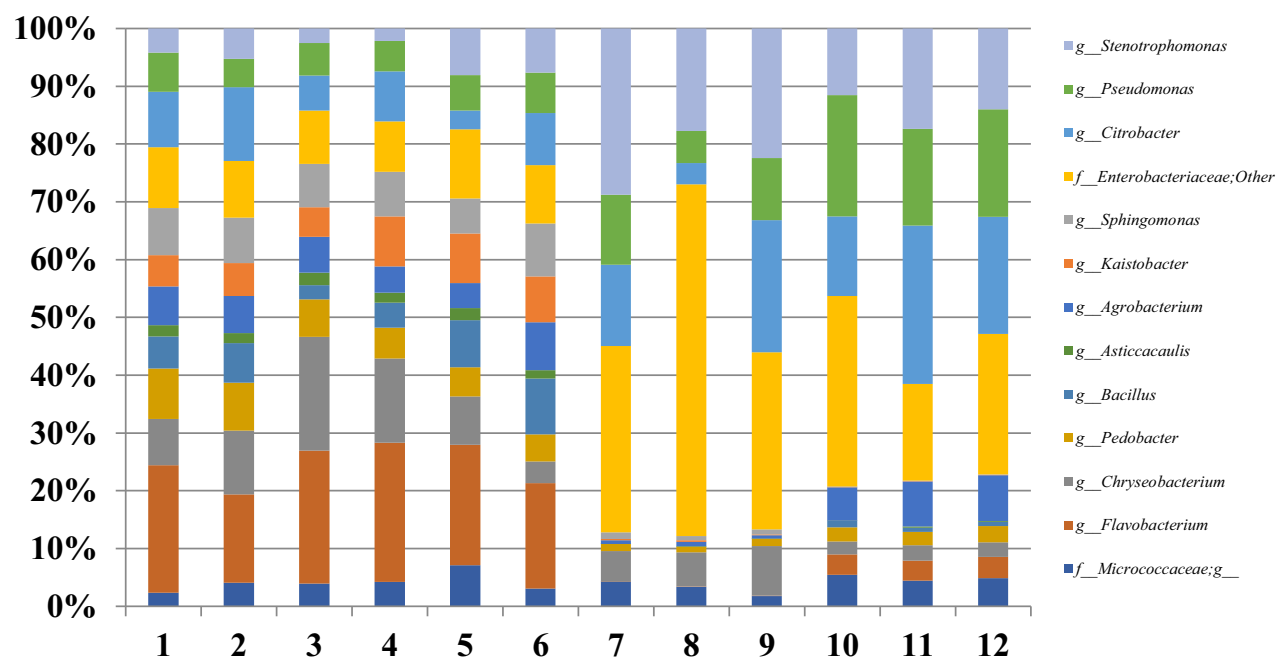

Fig. 1. Representation of the main bacterial genera in samples of the rhizosphere (1-6) and rhizoplane (7-12) of Early Zhukovsky potato when cultivated on gray forest soil in the flowering phase of plants.

Thus, it is established that the structure of root bacterial communities of potato plants is extremely diverse and complex. In this case, if the qualitative composition of the bacterial microbiota of the rhizosphere and rhizoplane is similar, there are significant differences in the ratio of certain groups of bacteria in these ecological niches, indicating a significant influence of plants on the formation of microbiota in different compartments. Studies of the diversity of the microflora of the rhizosphere and rhizoplane, the patterns of their formation, and the influence of various factors on their quantitative and qualitative composition are important for a deeper understanding of the role of associative bacteria in the productivity of such an important crop as potatoes.

\section{Acknowledgment}

The work is executed with financial support of grant RFBR-20-316-90047.

\section{References}

1. K. Schlaeppi, D. Bulgarelli, Mol Plant-Microbe Interact, 25 (2015)

2. L. D. Lopes, E. Pereira, C. Silva Mde, F. D. Andreote, Front. Microbiol, 7 (2016)

3. V. Venturi, C. Keel, Trends Plant Sci., 21 (2016)

4. L. Philippot, J. M. Raijmakers, P. Lemanceau, W. H. Putten, Nat. Rev. Microbiol, 11 (2013)

5. L.W. Mendes, E. E. Kuramae, A. A. Navarrete, J. A. van Veen, S.M. Tsai, ISME. 8 (2014)

6. A. Tkacz, P. Poole, J. Exp. Bot., 66, 8 (2015)

7. N. Wang, T. Jin, P. Trivedi, J.C. Setubal, J. Tang, A. et al., J Citrus Pathol, 2, 1 (2015) 
8. P. Vandenkoornhuyse, A. Quaiser, M. Duhamel, A. Le Van, A. Dufresne, New Phytologist, 206, 4 (2015)

9. G. Berg, M. Grube, M. Schloter, K. Smalla, Front Microbiol., 5, 148 (2014)

10. K. Farrar, D. Bryant, N. Cope-Selby // Plant Biotechnol J. 12, 9 (2014)

11. M. Khan, A. Sessitsch, M. Harris, K. Fatima, A. Imran, M. Arslan, G. Shabir, M. Qaiser, M. Khan, M. Afzal, Front Plant Sci., (2015)

12. S. Hacquard, S. Spaepen, R. Garrido-Oter, P. Schulze-Lefert, Annu. Rev. Phytopathol, 55 (2017)

13. Y. Yang, N. Wang, X. Guo, Y. Zhang, B.Ye, Plos one. (2017)

14. X. Zhang, R. Zhang, J. Gao, X. Wang, F. Fan, X. Ma, et al., Soil Biol. Biochem, 104 (2017)

15. L. Mommer, J. Kirkegaard, J. van Ruijven, Trends Plant Sci., 21 (2016)

16. A. Rosier, U. Bishnoi, V. Lakshmanan, Plant Mol. Biol., 90 (2016)

17. J. Sasse, E. Martinoia, T. Northen, Trends Plant Sci., 23 (2017)

18. D. V. Badri, J.M. Chaparro, R. Zhang, Q. Shen, J. M. Vivanco, J. Biol. Chem, 288 (2013)

19. J. Edwards, C. Johnson, C. Santos-Medellı'n, E. Lurie, N. K. Podishetty, S. Bhatnagar, et al., Proc Natl Acad Sci USA., 112, 8 (2015)

20. Jenkins S.N., Waite I.S., Blackburn A., Husband R., Rushton S. P., Manning D.C., et al, Antonie Van Leeuwenhoek., 95 (2009)

21. Fierer N., Bradford M.A., Jackson R.B, Ecology, 88 (2007) 